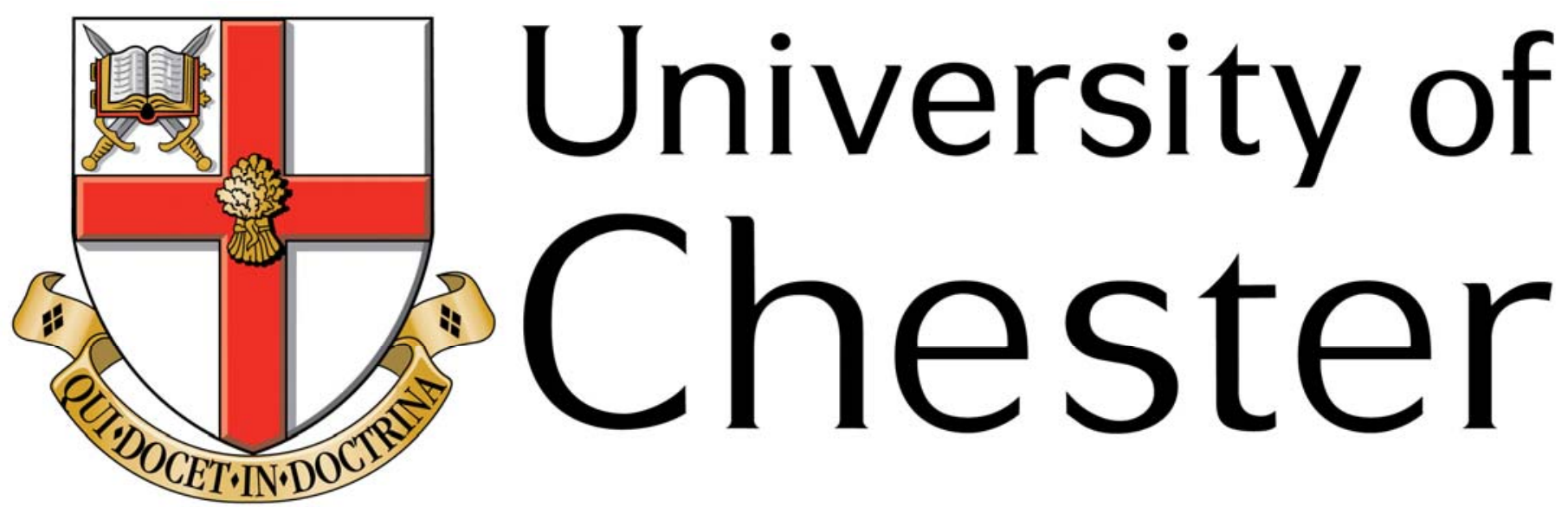

This work has been submitted to ChesterRep - the University of Chester's online research repository

\title{
http://chesterrep.openrepository.com
}

Author(s): Neville J Ford; Patricia M Lumb

Title: Mixed-type functional differential equations: A numerical approach

Date: 2009

Originally published in: Journal of Computational and Applied Mathematics

Example citation: Ford, N. J., \& Lumb, P. M. (2009). Mixed-type functional differential equations: A numerical approach. Journal of Computational and Applied Mathematics, 229(2), 471-479

Version of item: Author's preprint

Available at: http://hdl.handle.net/10034/209950 


\title{
Mixed-type functional differential equations: a numerical approach
}

\author{
Neville $\mathrm{J}_{\text {Ford }}{ }^{1}$ and Patricia M Lumb \\ Mathematics Department, University of Chester, Parkgate Road, Chester, CH1 \\ $4 B J, U K$
}

\begin{abstract}
The equations considered in this paper are mixed-type functional equations (sometimes known as forward-backward differential equations) that take the form

$$
u^{\prime}(t)=a u(t)+b u(t-1)+c u(t+1), t \in I \subset \mathbb{R} .
$$

We consider basic existence and uniqueness properties when $I=\left[t_{1}, t_{2}\right]$ and we seek solutions $u \in C\left[t_{1}-1, t_{2}+1\right]$ that satisfy

$$
u(t)=w_{1}(t), t \in\left[t_{1}-1, t_{1}\right], \quad u(t)=w_{2}(t), t \in\left[t_{2}, t_{2}+1\right],
$$

for prescribed functions $w_{1}, w_{2}$ absolutely continuous, respectively, on $\left[t_{1}-1, t_{1}\right],\left[t_{2}, t_{2}+\right.$ 1]. With arbitrary boundary conditions specified in this way, the problem turns out to be ill-posed and so existence and uniqueness questions have an important role to play in developing numerical schemes.

We consider, with $t_{1}, t_{2} \in \mathbb{N}$, numerical approximations of a solution when it exists. The numerical methods that we consider are linear $\theta$-methods and we investigate computationally their effectiveness through some illustrative examples. Keywords: delay equations, advanced equations, mixed functional differential equations, numerical methods
\end{abstract}

AMS subject classification: 34K06,34K28,65Q05

\section{Introduction and background information}

Functional differential equations with both delayed and advanced arguments are generally referred to as mixed (type) functional differential equations (MFDEs) or forward-backward equations. The study of these equations was

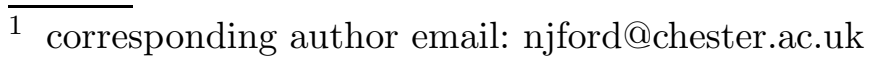


originally motivated by optimal control problems (see [13]) but applications also arise in other areas. We highlight example equations arising in nerve conduction theory (in [2]), economic dynamics (in [14]) and travelling waves in a spatial lattice (in [1])). In many example applications, the independent variable is likely to be spatial rather than temporal.

The analysis of MFDEs presents a significant challenge and it is appropriate for us to devote some space in this paper gathering together various insights and results. Our aim is to provide sufficient analytical background on which to build the numerical scheme that we shall discuss later.

To be precise, we shall investigate solutions to equations of the form:

$$
u^{\prime}(t)=a u(t)+b u(t-1)+c u(t+1), t \in I \subset \mathbb{R} .
$$

One might reasonably expect to gain some insights into these problems from the existing literature on retarded differential equations of the form

$$
u^{\prime}(t)=a u(t)+b u(t-1)
$$

(the case $c=0$ ) and these insights will be our starting point in this paper. Further insights could be obtained from the advanced equation $(b=0)$.

\section{MFDEs as boundary value problems}

We are accustomed to needing to specify initial or boundary conditions for a differential equation in order to be able to arrive at a unique solution. Therefore a natural starting point is to consider the appropriate form of conditions that will be needed for a MFDE of the form (1). Our insights from the study of retarded and advanced differential equations suggest that the conditions will be required in terms of function values over one or more intervals. To make things clearer, one can attempt to solve (1) by applying Laplace transforms. If we assume that a solution $u$ exists in a suitable space, satisfies (1) for $t \in I=\left[t_{1}, t_{2}\right]$ and that $u(t)=w_{1}(t), t \in\left[t_{1}-1, t_{1}\right], \quad u(t)=w_{2}(t), t \in$ $\left[t_{2}, t_{2}+1\right]$ and that $u$ is defined to be zero elsewhere, then we can show that the Laplace transform of the solution, if it exists, is given in terms of the boundary functions $w_{1}, w_{2}$.

Unfortunately, as we shall see later, the specification of such boundary functions is not sufficient to ensure that a solution can be found. It turns out that the underlying problem is both ill-posed (see section 3) and highly unstable (the characteristic quasi-polynomial has roots with both positive and negative real part). We shall discuss this further in the next sections. 


\section{$3 \quad$ Existing insights}

The ill-posedness of MFDEs is discussed by Rustichini [13], who described it as the "single most relevant difficulty encountered when dealing with MFDEs". Further evidence is readily found elsewhere in the existing literature. Consequences of the problem being ill-posed include the following:

- Linear equations with constant coefficients may not have a solution for given boundary conditions. For example, equation (1) with $a=0, b=1, c=1$ and initial condition $u_{0} \equiv 1$ has no everywhere continuous solution since $u(t)=(-1)^{k}$ is the only function (and is not continuous) that could possibly be a solution of this initial-value problem $[7,13]$.

- a variation of constants formula analogous to that for ODEs and retarded differential equations does not seem possible [14].

Further consequences are described in $[7,9,12]$.

The problem of ill-posedness has been avoided or overcome by several authors. Mallet-Paret and Verduyn Lunel decompose solutions as sums of 'forward' and 'backward' solutions in [12]. Rustichini ([14]) restricts the action of a linear operator of mixed type to functions which are periodic. This enables the operator to be identified with one of the delay type. Härterich et al ([7]) discuss finding functions $\varphi(t)$ for which a solution exists on either $\mathbb{R}^{+}$or $\mathbb{R}^{-}$. However, they comment on the difficulty of determining whether or not the set of eigenfunctions is complete. Verduyn Lunel (in [15]) gives conditions which guarantee completeness of the set of eigenfunctions for autonomous FDEs which links this work with our interest in super-exponentially decaying solutions for retarded differential equations (see $[4,5,10]$ ) but here there may be a further complication with the existence of super-exponentially growing solutions. Mallet-Paret established an existence theory for a class of MFDEs of mixed type using a linear Fredholm theory and the implicit function theory (see [11]). Collard et al. (in [3]) develop and implement a numerical procedure 'to solve for the short run dynamics of a neoclassical growth model with a simple time-to-build lag'. (They use a Runge-Kutta type of algorithm combined with a shooting method). Abell et al. have developed a collocation code, COLMTFDE, for MFDEs. We refer the reader to [1] for further details. Despite the attention to MFDEs in the literature since 1989, Abell et al ([1]) still refer, as recently as 2005, to the 'lack of analytical techniques and numerical solvers for differential equations with both forward and backward delays'. 


\section{The characteristic quasi-polynomial}

Many of the interesting features and challenges of MFDEs become apparent when one considers the roots of the characteristic quasi-polynomial which, for (1), takes the form

$$
\lambda=a+b e^{-\lambda}+c e^{\lambda} .
$$

If $\lambda$ satisfies (3) then $u(t)=e^{\lambda t}$ satisfies (1) on any interval $I \subset \mathbb{R}$. The values $\lambda$ are known as characteristic values, or eigenvalues, of (1) and the corresponding functions $e^{\lambda t}$ are the characteristic functions or eigenfunctions. By setting $\lambda=x+i y, x, y \in \mathbb{R}$ and $x \neq \frac{1}{2} \ln \left|\frac{b}{c}\right|$. We obtain

$$
\begin{gathered}
\cos y=\frac{(x-a)}{\left(b e^{-x}+c e^{x}\right)}, \\
\sin y=\frac{y}{\left(-b e^{-x}+c e^{x}\right)},
\end{gathered}
$$

which can be combined to give a more convenient equation

$$
y= \pm\left(c e^{x}-b e^{-x}\right) \sqrt{1-\frac{(x-a)^{2}}{\left(b e^{-x}+c e^{x}\right)^{2}}} .
$$

In the figures that follow, we consider the equation

$$
u^{\prime}(t)=2 u(t)-5 u(t-1)+3 u(t+1) .
$$

In Figure 1 we plot the graphs of $y$ against $x$ given by (4) and (6). In Figure 2 we plot the corresponding graphs for $\cos (y)$ against $x$. The characteristic values $\lambda$ will be given by the values of $x, y$ corresponding to points where the graphs given by (4) and (6) intersect. Of course, we had to choose values for the constants, but one obtains similar graphs for any choice of non-zero constants we tried.

Interpreting the graphs, we can see that there are infinitely many characteristic values with positive $x$ co-efficient and infinitely many with negative $x$ coefficient. The characteristic roots do not have any cluster points. In the special cases where either $b=0$ or $c=0$, either the left hand or right hand branch of one of the graphs is absent which accords with our previous experience for retarded differential equations.

The implication of these characteristic values is significant. There are eigenfunctions with arbitrarily large exponential growth rates and eigenfunctions with arbitrarily large exponential decay rates. This means that, whether we project forward in time from the left hand end of the interval or backwards 
in time from the right hand end, the solution we obtain will be arbitrarily highly unstable with respect to small changes in the boundary function. This explains the possible motivation for decomposing the solution into forward and backward components.

For a given $a, b, c$ we can obtain a value $x=x_{1}$ and find $y_{1}$ such that $\lambda=x_{1}+$ $i y_{1}$ is a characteristic value and hence plot a particular eigenfunction for the equation. In Figures 3 and 4 we illustrate typical solutions, for eigenfunctions with $x_{1}<0$ and $x_{1}>0$ respectively. In each case the same equation is used for all four graphs but the time period over which the solution is given varies.

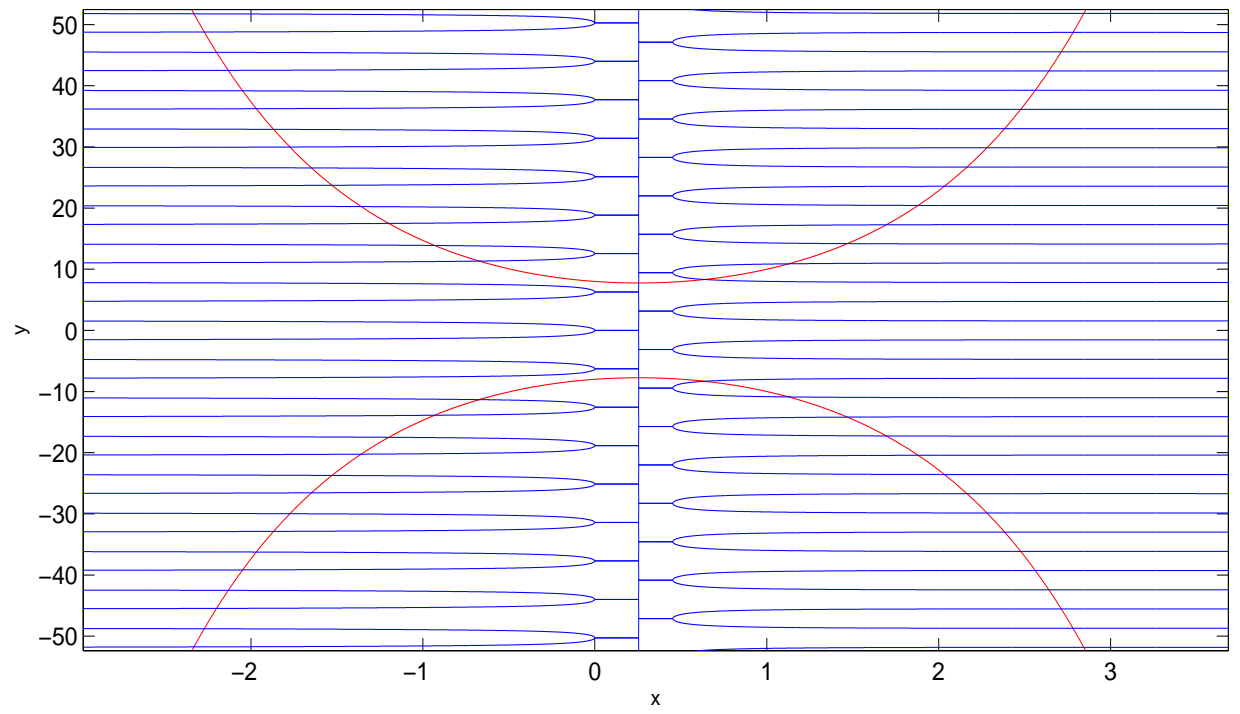

Fig. 1. $y$ against $x$ given by (4) and (6) for (7)

\section{Existence and uniqueness of solutions}

The previous section has illustrated some of the difficulties in developing theoretical results for MFDEs. Now we move on to develop some theory. First we clarify what is meant by a solution of a MFDE. Definition 5.1 is given by Mallet-Paret and Verduyn Lunel. See $[9,13]$ for alternative definitions

Definition 5.1 [12] A solution of equation (1) on an interval $\left[t_{1}, t_{2}\right] \subseteq \mathbb{R}$ is a continuous function $x:\left[t_{1}-1, t_{2}+1\right] \rightarrow \mathbb{C}$ which is absolutely continuous on $\left[t_{1}, t_{2}\right]$ and which satisfies equation (1) for almost every $t \in\left[t_{1}, t_{2}\right]$.

Next we remark that a simple transformation of MFDEs is possible. We use the substitution $\tilde{u}(t)=e^{a t} u(t)$ to transform (1) into an equation of the form

$$
\tilde{u}^{\prime}(t)=\alpha \tilde{u}(t+1)+\beta \tilde{u}(t-1), t \in\left[t_{1}, t_{2}\right]
$$




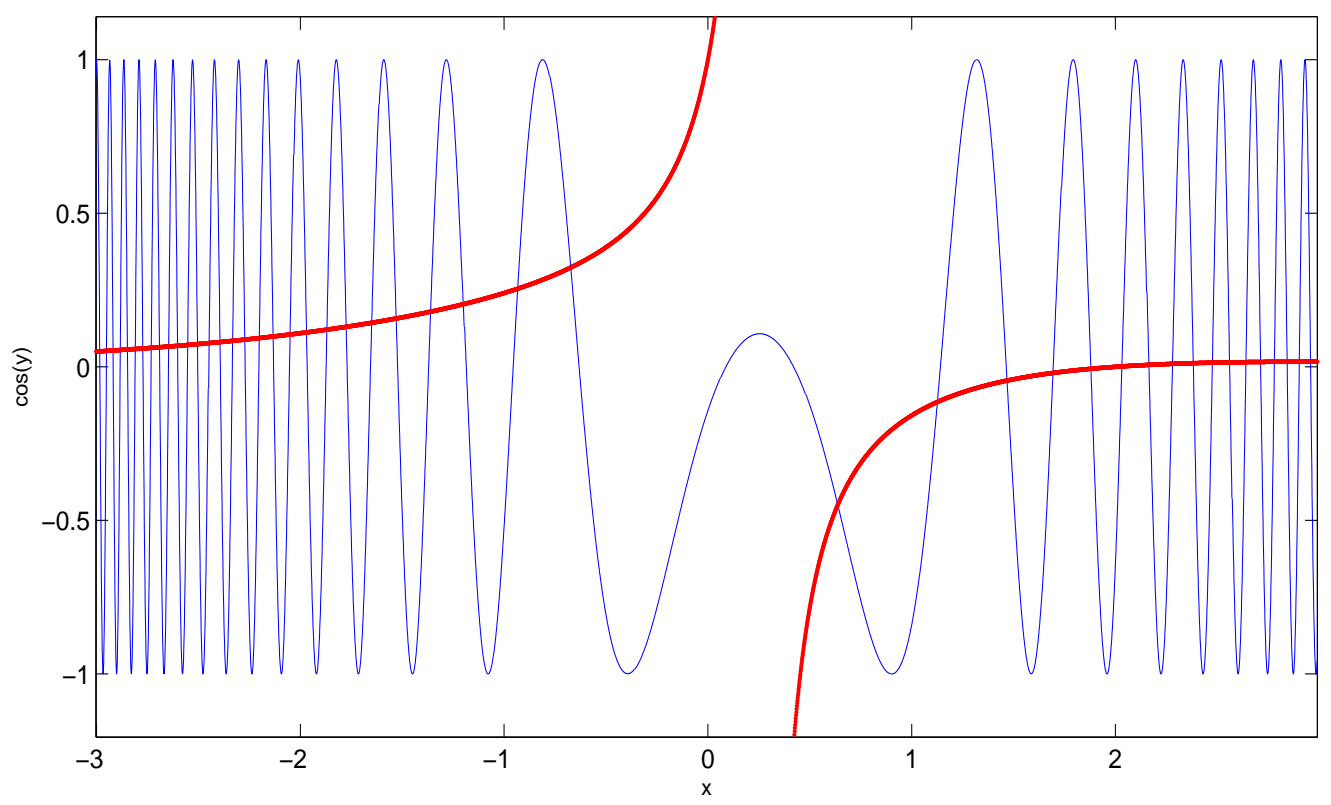

Fig. 2. $\cos (y)$ against $x$ given by (4) and (6) for (7)
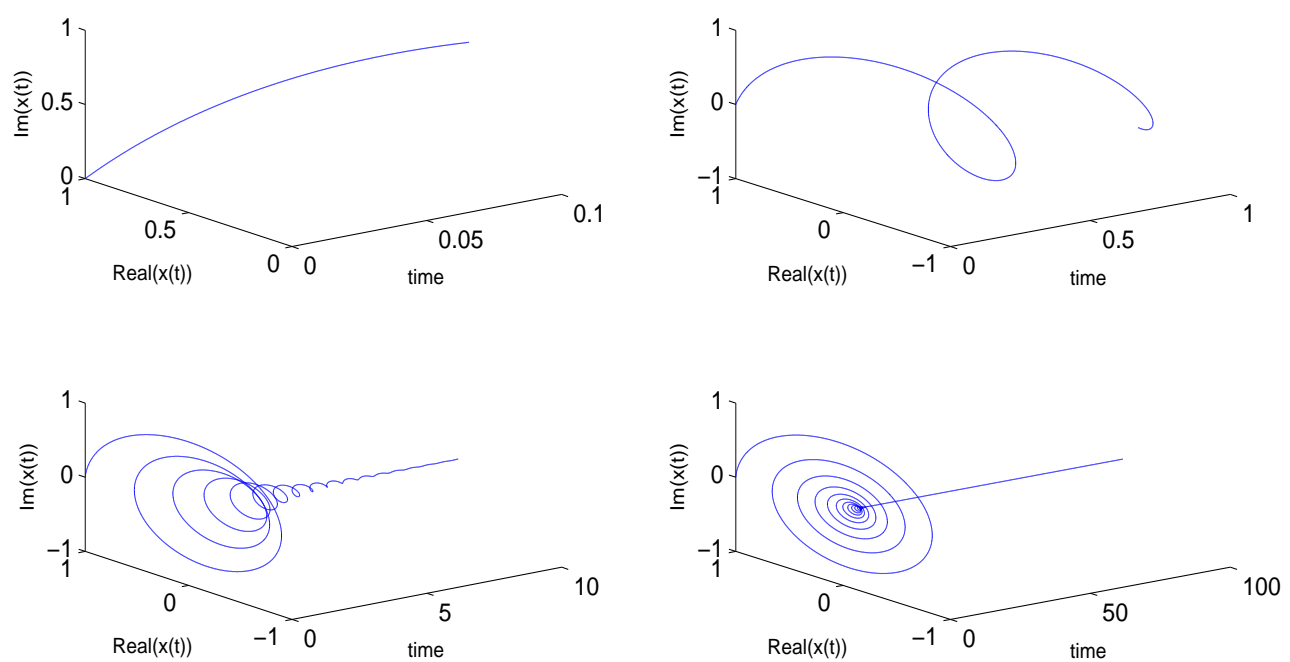

Fig. 3. Typical solutions for (7) $\quad x_{1}=-0.6723$

We are thus able to focus our attention on equations that do not include an instantaneous term. 

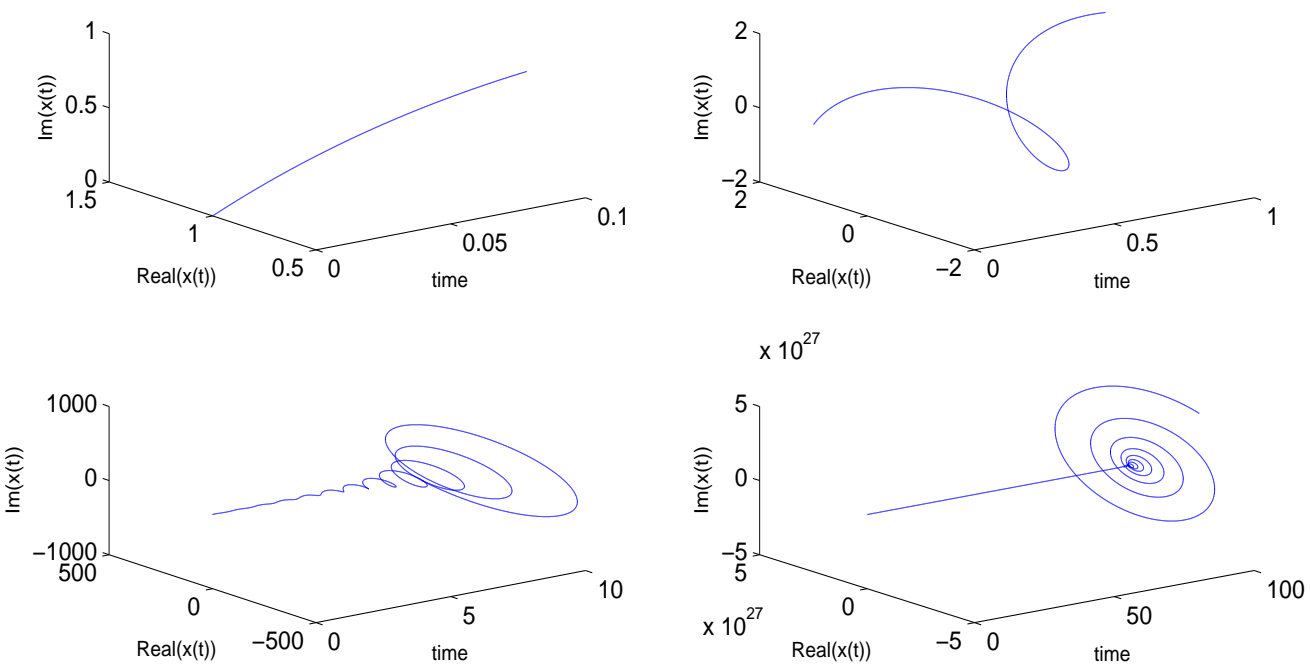

Fig. 4. Typical solutions for (7) $\quad x_{1}=0.6379$

We follow the methodology adopted in [8] to extend the solution to the right. We rewrite (8) in the form

$$
u(t)=a u^{\prime}(t-1)+b u(t-2) \text { where } a=\frac{1}{\alpha}, \quad b=-\frac{\beta}{\alpha}, \quad t \in\left[t_{1}+1, t_{2}+1\right] .
$$

To establish an existence theorem, we suppose $\alpha \neq 0, t_{1}=0, t_{2}=k-1 \in \mathbb{N}$ and treat (9) as an initial value problem. with the initial conditions $u(t)=\varphi(t)$, $t \in[-1,1]$, with

$$
\varphi(t)=\left\{\begin{array}{l}
\varphi_{1}(t) \text { for } t \in[-1,0] \\
\varphi_{2}(t) \text { for } t \in(0,1] .
\end{array}\right.
$$

Table 1 gives the solution using the method of steps for $u(t)$ for $t \in(1,5]$. $u(t)=\varphi(t), t \in[-1,1]$, where

\begin{tabular}{|c|l|}
\hline Interval for $t$ & Solution $x(t)$ \\
\hline$(1,2]$ & $a \varphi_{2}^{\prime}(t-1)+b \varphi_{1}(t-2)$ \\
\hline$(2,3]$ & $a^{2} \varphi_{2}^{\prime \prime}(t-2)+b \varphi_{2}(t-2)+a b \varphi_{1}^{\prime}(t-3)$ \\
\hline$(3,4]$ & $a^{3} \varphi_{2}^{\prime \prime \prime}(t-3)+2 a b \varphi_{2}^{\prime}(t-3)+a^{2} b \varphi_{1}^{\prime \prime}(t-4)+b^{2} \varphi_{1}(t-4)$ \\
\hline$(4,5]$ & $a^{4} \varphi_{2}^{(4)}(t-4)+3 a^{2} b \varphi_{2}^{\prime \prime}(t-4)+b^{2} \varphi_{2}(t-4)+a^{3} b \varphi_{1}^{\prime \prime \prime}(t-5)$ \\
& $+2 a b^{2} \varphi_{1}^{\prime}(t-5)$ \\
\hline
\end{tabular}

Table 1

Solution to (9) over successive intervals 
Continuing in this way we find that for $t \in(2 \ell-1,2 \ell], \ell \in \mathbb{N}$ the solution $u(t)$ is given by

$u(t)=\Sigma_{k=0}^{\ell-1} \gamma_{\ell, 2 k} a^{2 k} b^{\ell-k} \varphi^{(2 k)}(t-2 \ell)+\Sigma_{k=0}^{\ell-1} \gamma_{\ell, 2 k+1} a^{2 k+1} b^{\ell-k-1} \varphi^{(2 k+1)}(t-(2 \ell-1))$

and for $t \in(2 \ell, 2 \ell+1]$ by

$u(t)=\Sigma_{k=0}^{\ell} \delta_{\ell, 2 k} a^{2 k} b^{\ell-k} \varphi^{(2 k)}(t-2 \ell)+\Sigma_{k=0}^{\ell-1} \delta_{\ell, 2 k+1} a^{2 k+1} b^{\ell-k} \varphi^{(2 k+1)}(t-(2 \ell+1))$,

where $\gamma_{v, w}$ and $\delta_{v, w}, v, w \in \mathbb{N}$, are defined for $\ell \geq 1$ and $v \leq 2 \ell-1$ as follows:

$$
\begin{aligned}
\gamma_{\ell, 2 k} & =\sum_{i=0}^{k} \gamma_{\ell-k-1+i, 2 i}+\sum_{i=0}^{k-1} \delta_{\ell-k-1+i, 2 i+1} \\
\gamma_{\ell, 2 k+1} & =\sum_{i=0}^{k} \gamma_{\ell-k-1+i, 2 i}+\sum_{i=0}^{k} \delta_{\ell-k-1+i, 2 i+1} \\
\delta_{\ell, 2 k} & =\gamma_{\ell+1,2 k} \\
\delta_{\ell, 2 k+1} & =\gamma_{\ell, 2 k+1} .
\end{aligned}
$$

We can show that $\gamma_{\ell, 0}=1, \gamma_{\ell, 2 \ell-1}=1, \gamma_{\ell, 2 \ell-2}=1, \delta_{\ell, 0}=1, \delta_{\ell, 2 \ell-1}=1$, $\delta_{\ell, 2 \ell}=1$, and establish the following relationships

$$
\begin{aligned}
\gamma_{p, 2 k-1}+\gamma_{p, 2 k} & =\gamma_{p+1,2 k} \\
\gamma_{p+1,2 k}+\gamma_{p, 2 k+1} & =\gamma_{p+1,2 k+1}
\end{aligned}
$$

We note that $\gamma_{v, w}=\delta_{v, w}=0$ for $v<0$ and that $\gamma_{\ell, w}=\delta_{\ell, w}=0$ for $j>2 \ell-1$. We can prove the validity of expressions (11) and (12) by induction (see the Technical Report [6] for the proofs of this and the subsequent Theorems).

Remark 5.1 The solution can be extended to the left in a similar manner by rewriting the equation in the form $u(t)=a u^{\prime}(t+1)+b u(t+2)$. Of course, in that case we would need boundary conditions defined on $[k-2, k]$.

Necessary and sufficient conditions for the solution given by (11) and (12) to be continuously differentiable are given by the following Theorem. Notice that, contrary to experience with retarded differential equations, here the solutions become less smooth rather than more smooth as the solution propagates.

Theorem 5.1 Define $u(t)$ as in equations (11)and (12).

For $m=1,2, \ldots, k, u\left(m^{+}\right)=u\left(m^{-}\right)$and $u^{\prime}\left(m^{+}\right)=u^{\prime}\left(m^{-}\right)$ if and only if $\varphi^{(n+1)}(0)=\alpha \varphi^{(n)}(1)+\beta \varphi^{(n)}(-1)$ for $n=0,1, \ldots, k$.

This result can be extended under further conditions on the initial function to guarantee that the solution is in $\mathcal{C}^{\infty}$.

Theorem 5.2 The solution to equation (8) with $\varphi \in \mathbb{C}_{[-1,1]}^{\infty}$ exists and is differentiable if and only if $\varphi^{(n+1)}(0)=\alpha \varphi^{(n)}(1)+\beta \varphi^{(n)}(-1)$ for $n=0,1,2, \ldots$. 
Under the conditions of Theorem 5.1 or Theorem 5.2 we have the following uniqueness Theorem:

Theorem 5.3 Let $\varphi \in \mathbb{C}_{[-1,1]}^{\infty}$, then there is at most one differentiable function $u(t)$ that satisfies equation (8) with $u(t)=\varphi(t)$ for $t \in[-1,1]$.

Remark 5.2 As is familiar from the treatment of retarded differential equations, discontinuities in the solution may arise at the integer points if no conditions, such as those in Theorem 5.1, are imposed on $\varphi$.

\section{Numerical methods}

It would be very easy to construct a numerical method for providing an approximate solution to an equation of the form (9) subject to initial conditions defined on $[-1,1]$. However, we are interested in solving (1) or (2) subject to the boundary conditions given on $[-1,0]$ and $[k-1, k]$ for some $k \in \mathbb{N}$. Our approach will be to use the boundary conditions to provide approximate initial conditions on $[-1,1]$ and, in turn, to solve the initial value problem for a solution on $[1, k-1]$.

To be precise, we now imagine the case of $(10)$ where $\varphi_{1}(t)$ is known, $\varphi_{2}(t)$ is unknown but $u(t)$ is known to equal $f(t)$ for $t \in[k-1, k]$.

We introduce the following notation: We fix $N \in \mathbb{N}, h>0$ and define

$$
y_{n+N}=\left(x_{n+N} x_{n+N-1} \ldots \ldots x_{n+1} x_{n} \ldots \ldots x_{n-N}\right)^{T}
$$

where $x_{j} \approx u(j h)$. We note that $x_{-N}, \ldots, x_{0}$ can be obtained from the known values of $\varphi_{1}(t)$ while $x_{1}, \ldots, x_{N}$ should be obtained from the unknown function $\varphi_{2} . x_{(k-1) N+1}, \ldots, x_{k N}$ may be obtained from values of the function $f$.

We apply a linear $\theta$ - method to (1), with the fixed step length $h=\frac{1}{N}$, and this leads to an equation of the form

$$
y_{n+N}=A y_{n+N-1} \text { with } A=\left(\begin{array}{cc}
M_{1} & M_{2} \\
M_{3} & M_{4}
\end{array}\right)
$$

where $M_{1}$ takes the form $\left(-\frac{(1-\theta)}{\theta} 0 \ldots 0 \frac{(1-h \theta a)}{h \theta c} \frac{-[1+h(1-\theta) a]}{h \theta c} 0 \ldots 0 \frac{-b}{c}\right), M_{2}=$ $\left(\frac{-b(1-\theta)}{\theta c}\right), M_{3}$ is an appropriate identity matrix and $M_{4}=(0 \ldots . .0)^{T}$. Since $y_{n+N}=A y_{n+N-1}=A^{2} y_{n+N-2}=A^{3} y_{n+N-3}=\ldots$. , it follows that $y_{n+N}=A^{N} y_{n}$ and $y_{n+2 N}=A^{2 N} y_{n}, y_{n+3 N}=A^{3 N} y_{n}, \ldots, y_{n+k N}=A^{k N} y_{n}$. 
Thus $y_{k N}=A^{(k-1) N} y_{N}$ which leads to an equation of the form

$$
\left(\begin{array}{l}
F \\
*
\end{array}\right)=\left(\begin{array}{ll}
D & B \\
* & *
\end{array}\right)\left(\begin{array}{l}
X \\
C
\end{array}\right)
$$

where $X \in \mathbb{R}^{N \times 1}$, is an unknown vector containing the values $x_{1}, \ldots, x_{N}$ and the matrices $B \in \mathbb{R}^{N \times(N+1)}, C \in \mathbb{R}^{(N+1) \times 1}, D \in \mathbb{R}^{N \times N}$ and $F \in \mathbb{R}^{N \times 1}$. It turns out that we do not need to concern ourselves with matrix entries marked $*$. For a particular problem $F, D, B$ and $C$ will be known and the important equation will be $F=D X+B C$. We aim to use this equation to find $X$, a set of approximations to values of $\varphi_{2}$ on $[0,1]$. By varying $N$ we can choose the density of approximations. Of course, $k$ will be fixed in a particular example, but we can perform the same technique for any natural number $k$.

Finally, we use the function $\varphi_{1}$ on $[-1,0]$ together with the values contained in the vector $X$ to provide the starting vector, $y_{N}$, for the formula (19) which can be used to propagate the solution over successive unit intervals on $[0, k-1]$.

We are interested in establishing how good an approximation this method will provide to the exact solution to the MFDE on $[0, k]$. This is determined by the quality of the approximation of the solution at the points on $[0,1]$. Thus it makes sense for us to compare the computed sequence $X$ with the sequence $X_{E}$, derived from the exact solution with the same grid points for the chosen test problem. Of course we must choose test problems where the exact solution is known on $[0, k]$.

\section{Numerical examples}

In each example we use equations with a known exact solution and use the conditions that $x(t)$ is equal to this function on $[-1,0]$ and on $(k-1, k]$. We discretise the equation and, following the method outlined in Section 6, obtain a solution on $(0,1]$. We estimate the 2 -norm of the error by finding

$$
\mathcal{E}=h \times \|\left(X-X_{E} \|_{2} .\right.
$$

The values of $\mathcal{E}^{2}$ are tabulated for the examples that follow and enable us to estimate the order of convergence, $p$, as $h \rightarrow 0$ of $X$ to $X_{E}$.

Example 7.1 We consider $x^{\prime}(t)=\frac{1}{2} x(t+1)-\frac{1}{2} x(t-1)$ on $(0,1]$ given $\varphi_{1}(t)=$ $t^{2}, t \in[-1,0]$, and $f(t)=t^{2}, t \in[3,4]$.

In Table 2 we present values of $\mathcal{E}^{2}$ and estimates for the order $p$ for the forward and backward Euler methods and for the trapezium rule. We observe 
Table 2

\begin{tabular}{|l|l|c|c|c|c|c|}
\hline & & \multicolumn{4}{|c|}{$\mathcal{E}^{2}$ and estimates $\hat{p}$ for the order $p$} \\
\hline \multirow{2}{*}{$i$} & \multicolumn{1}{|c|}{$\begin{array}{c}\text { Step } \\
\text { length }\end{array}$} & \multicolumn{2}{|c|}{$\begin{array}{c}\text { Forward Euler } \\
\text { method }\end{array}$} & \multicolumn{2}{c|}{$\begin{array}{r}\text { Backward Euler } \\
\text { method }\end{array}$} & $\begin{array}{c}\text { Trapezium } \\
\text { rule }\end{array}$ \\
\cline { 2 - 7 } & $h_{i}$ & $\mathcal{E}^{2}$ & $\hat{p}$ & $\mathcal{E}^{2}$ & $\hat{p}$ & $\mathcal{E}^{2}$ \\
\hline 1 & 0.5 & 4.508574 & & 1.909368 & & 0 \\
2 & 0.25 & 1.721040 & 0.6947 & 0.398952 & 1.1294 & 0 \\
3 & 0.125 & 0.528169 & 0.8521 & 0.086295 & 1.1044 & 0 \\
4 & 0.0625 & 0.145308 & 0.9309 & 0.019675 & 1.0665 & 0 \\
5 & 0.03125 & 0.038016 & 0.9672 & 0.004670 & 1.0374 & 0 \\
6 & 0.015625 & 0.009716 & 0.9841 & 0.001136 & 1.0197 & 0 \\
7 & 0.0078125 & 0.002455 & 0.9923 & 0.000280 & 1.0102 & 0 \\
8 & 0.00390625 & 0.000617 & 0.9962 & 0.000069 & 1.0104 & 0 \\
\hline
\end{tabular}

Equation: $x^{\prime}(t)=\frac{1}{2} x(t+1)-\frac{1}{2} x(t-1)$ given $\varphi_{1}(t)=t^{2}, t \in[-1,0]$, and $f(t)=$ $t^{2}, t \in[3,4] . \mathcal{E}^{2}=h^{2}\left\|X-X_{E}\right\|_{2}^{2}$ on $[0,1]$ and estimates of order $p$.

that $p \rightarrow 1$ as $h \rightarrow 0$ for both Euler methods. The trapezium rule is exact for this problem.

We now consider equation (20), which has as an exact solution $x(t)=e^{\alpha t}$, when $\varphi_{1}(t)$ and $f(t)$ both equal $e^{\alpha t}$. We present two examples, each time using the trapezium rule to discretise the equation

$$
x^{\prime}(t)=\left(\alpha-b e^{-\alpha}-c e^{\alpha}\right) x(t)+b x(t-1)+c x(t+1)
$$

Example 7.2 We consider equation (20) with $\alpha=1$ and with $k=2,3$ and 4 . In Table 3 we present the values of $\mathcal{E}^{2}$ and estimates for the order $p$. Results are as expected (with an order 2 observed for the trapezium rule) until the method becomes unreliable when the combination of small $h$ and larger $k$ leads to a nearly singular matrix. The values $*$ in the table correspond to those entries where Matlab reports that the results will be unreliable.

Example 7.3 We consider equation (20) for different values of $\alpha$. In Table 4 we present the values of $\mathcal{E}^{2}$ and estimates of $p$ for three values of $\alpha$. Again, we observe that the numerical method achieves its order providing that the matrix involved in the computation is not close to being singular.

Remark 7.1 We have seen that the results in our tables are limited, for small step lengths $h$, by the fact that the matrices in our computations may become close to singular. This is a limitation of our existing method (which is designed to provide a prototype algorithm) and could be avoided by the use of more 


\begin{tabular}{|c|l|c|c|c|c|c|c|}
\hline & & \multicolumn{5}{|c|}{$\mathcal{E}^{2}$ and estimates $\hat{p}$ of order $p$} \\
\hline \multirow{2}{*}{$\mathrm{i}$} & Step length & \multicolumn{2}{|c|}{$\mathrm{k}=2$} & \multicolumn{2}{c|}{$\mathrm{k}=3$} & \multicolumn{2}{c|}{$\mathrm{k}=4$} \\
\cline { 2 - 8 } & $h_{i}$ & $\mathcal{E}^{2}$ & $\hat{p}$ & $\mathcal{E}^{2}$ & $\hat{p}$ & $\mathcal{E}^{2}$ & $p$ \\
\hline 2 & 0.5 & $6.024 \times 10^{-4}$ & & $2.998 \times 10^{-3}$ & & $8.069 \times 10^{-3}$ & \\
2 & 0.25 & $2.449 \times 10^{-5}$ & 2.310 & $1.234 \times 10^{-4}$ & 2.301 & $3.332 \times 10^{-4}$ & 2.299 \\
3 & 0.125 & $1.193 \times 10^{-6}$ & 2.180 & $6.068 \times 10^{-6}$ & 2.173 & $1.644 \times 10^{-5}$ & 2.171 \\
4 & 0.0625 & $6.516 \times 10^{-8}$ & 2.097 & $3.332 \times 10^{-7}$ & 2.093 & $9.047 \times 10^{-7}$ & 2.092 \\
5 & 0.03125 & $3.796 \times 10^{-9}$ & 2.051 & $1.947 \times 10^{-8}$ & 2.049 & $5.293 \times 10^{-8}$ & 2.048 \\
6 & 0.015625 & $2.288 \times 10^{-10}$ & 2.026 & $1.176 \times 10^{-9}$ & 2.025 & $3.200 \times 10^{-9}$ & 2.024 \\
7 & 0.0078125 & $1.404 \times 10^{-11}$ & 2.013 & $7.222 \times 10^{-11}$ & 2.013 & $*$ & $*$ \\
8 & 0.00390625 & $8.7 \times 10^{-13}$ & 2.006 & $4.48 \times 10^{-12}$ & 2.005 & $*$ & $*$ \\
\hline
\end{tabular}

Table 3

(Trapezium Rule) Equation: $x^{\prime}(t)=\left(1-0.5 e^{-1}-0.5 e^{1}\right) x(t)+0.5 x(t-1)+0.5 x(t+1)$ given $\varphi_{1}(t)=e^{t}, t \in[-1,0]$, and $f(t)=e^{t}, t \in[k-1, k]: \mathcal{E}^{2}=h^{2}\left\|X-X_{E}\right\|_{2}^{2}$ on $[0,1]$ and estimates of $p$.

sophisticated techniques from numerical linear algebra for the solution of the underlying systems of equations. This is beyond the scope of the present paper.

Finally, we consider the approximation of the solution over the subsequent interval $[1, k-1]$. In Figures 5 and 6 we illustrate the trajectories of the solution on $[0, k]$ for $k=2,3,4,5$. In Figures 5 and 6 we use step lengths $h=\frac{1}{50}$ and $\frac{1}{128}$ respectively. We can see the effect of the near singular matrices reflected in the trajectories because in these cases the original solution given over $[k-1, k]$ is not recovered accurately. As we remarked earlier, this particular problem could be overcome by the use of improved solution methods for the linear systems.

\section{Conclusions and limitations of the approach}

The results of the paper are very satisfactory in providing a reliable method for finding solutions to MFDEs where the boundary conditions are given. We need to return to the question of whether or not a solution to such a problem exists! As we remarked at the beginning of the paper, the boundary value problem of MFDEs is not well-understood, but it is known that such a problem often has no solution. As we saw in Theorems 5.2 and 5.3 even for the initial function problem (8), there are quite stringent conditions required on the initial function in order to ensure that the solution has certain properties, such as being continuously differentiable, for example. 


\begin{tabular}{|c|l|c|c|c|c|c|c|}
\hline & & \multicolumn{6}{|c|}{$\mathcal{E}^{2}$ and estimates $\hat{p}$ of order $p$} \\
\hline \multirow{2}{*}{$\mathrm{i}$} & Step length & $\alpha=-0.5$ & \multicolumn{2}{|c|}{$\alpha=0.6$} & \multicolumn{2}{|c|}{$\alpha=3$} \\
\cline { 2 - 8 } & $h_{i}$ & $\mathcal{E}^{2}$ & $\hat{p}$ & $\mathcal{E}^{2}$ & $\hat{p}$ & $\mathcal{E}^{2}$ & $p$ \\
\hline 1 & 0.5 & $6.226 \times 10^{-7}$ & & $1.291 \times 10^{-4}$ & & 4.966 & \\
2 & 0.25 & $3.146 \times 10^{-8}$ & 2.153 & $5.627 \times 10^{-6}$ & 2.260 & $2.202 \times 10^{-1}$ & 2.248 \\
3 & 0.125 & $1.772 \times 10^{-9}$ & 2.075 & $2.863 \times 10^{-7}$ & 2.148 & $1.051 \times 10^{-2}$ & 2.195 \\
4 & 0.0625 & $1.052 \times 10^{-10}$ & 2.037 & $1.602 \times 10^{-8}$ & 2.080 & $5.589 \times 10^{-4}$ & 2.117 \\
5 & 0.03125 & $6.410 \times 10^{-12}$ & 2.018 & $9.454 \times 10^{-10}$ & 2.041 & $3.199 \times 10^{-5}$ & 2.063 \\
6 & 0.015625 & $4.000 \times 10^{-13}$ & 2.001 & $5.741 \times 10^{-11}$ & 2.021 & $1.910 \times 10^{-6}$ & 2.033 \\
7 & 0.0078125 & $*$ & $*$ & $*$ & $*$ & $1.166 \times 10^{-7}$ & 2.017 \\
8 & 0.00390625 & $*$ & $*$ & $*$ & $*$ & $6.863 \times 10^{-9}$ & 2.043 \\
\hline
\end{tabular}

Table 4

(Trapezium Rule) Equation: $x^{\prime}(t)=\left(\alpha-b e^{-\alpha}-c e^{\alpha}\right) x(t)+b x(t-1)+c x(t+1)$ given $\varphi_{1}(t)=e^{\alpha t}, t \in[-1,0]$, and $f(t)=e^{\alpha t}, t \in[3,4] . \mathcal{E}^{2}=h^{2}\left\|X-X_{E}\right\|_{2}^{2}$ on $[0,1]$ and estimates of $p$.
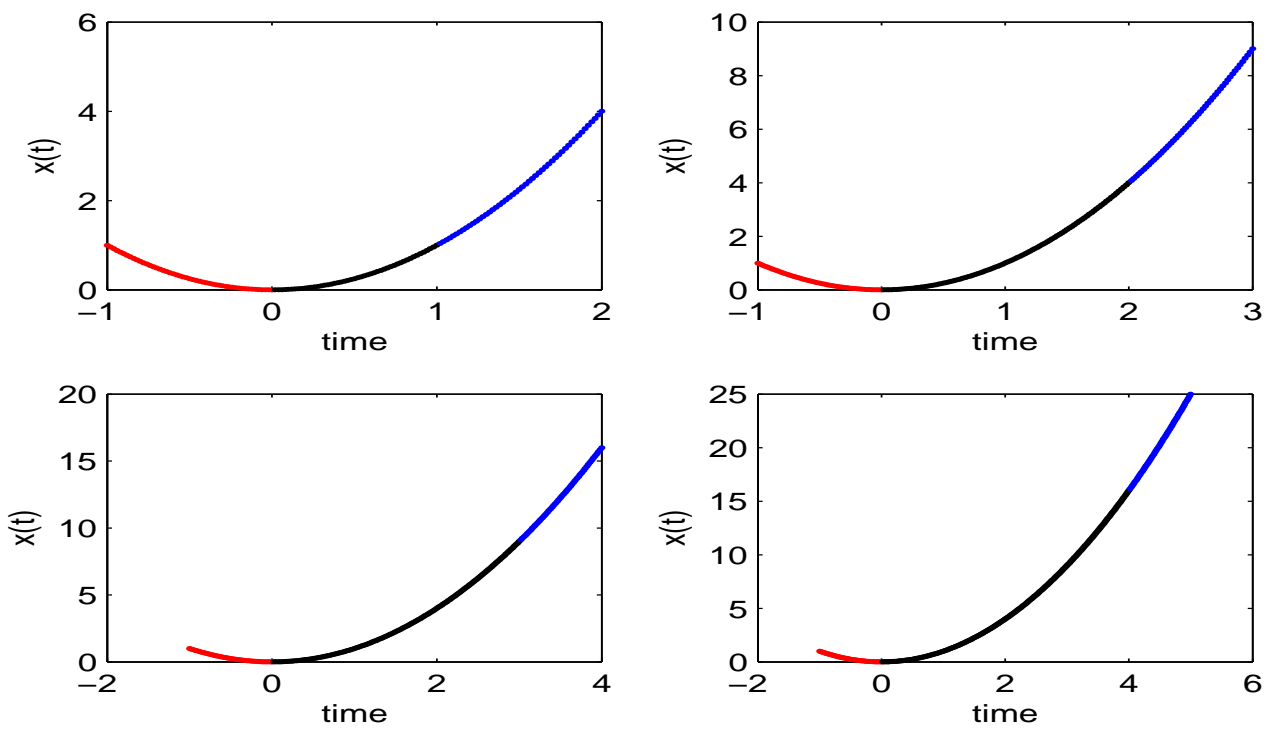

Fig. 5. See example 7.3: Trajectory of the approximate solution found on $[0, \mathrm{k}]$ for $\mathrm{k}=2,3,4,5 ; \mathrm{N}=50$

Given any set of boundary conditions and any MFDE of the form we considered here, the proposed numerical method will provide a solution. If the original problem had a continuously differentiable solution, then the solution found by the method in this paper is likely (assuming any problems with near singular matrices are circumvented) to be a good approximation to the true solution. However, if the original problem does not have a solution, then the numerical 

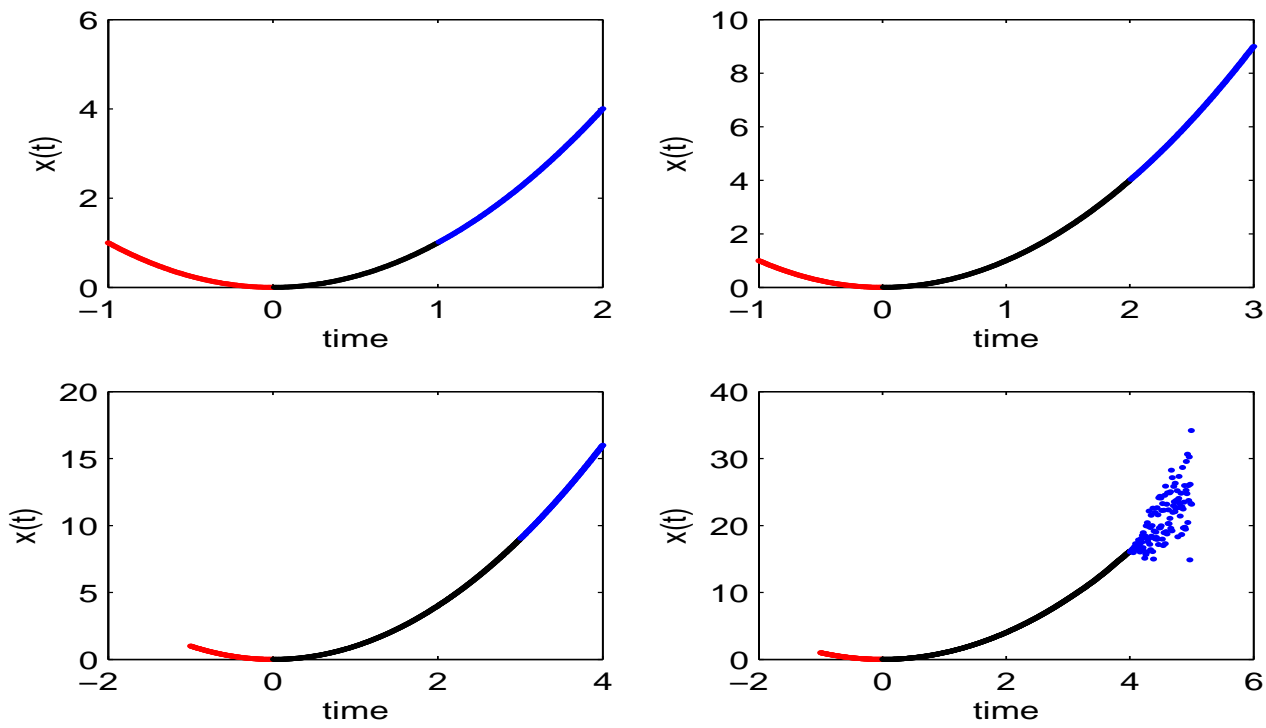

Fig. 6. See example 7.3: Trajectory of the approximate solution found on $[0, \mathrm{k}]$ for $\mathrm{k}=2,3,4,5 ; \mathrm{N}=128$

scheme will nevertheless provide a solution, but this time to a perturbation of the original problem! Caveat emptor! ${ }^{2}$

\section{Acknowledgements}

The authors are pleased to acknowledge the helpful comments of the referees and guest editor on the initial version of this paper.

\section{References}

[1] K. A. Abell, C. E. Elmer, A. R. Humphries, E. S. V. Vleck, Computation of mixed type functional differential boundary value problems, SIADS 4, (2005), 755-781.

[2] H. Chi, J. Bell, B. Hassard, Numerical solution of a nonlinear advance-delaydifferential equation from nerve conduction theory, J. Math. Biol. 24 (1986), 583-601.

$\overline{2}$ The warning Caveat emptor (let the buyer beware) is sometimes used in a cautionary way by computer software suppliers to indicate that the end-user must assume the ultimate responsibility for the reliability of the results provided by the software. 
[3] F. Collard, O. Licandro, L. .A. Puch, The short-run dynamics of optimal growth models with delays, Computing in Economics and Finance, 117, Society for Computational Economics (2004).

[4] N. J. Ford, P. M. Lumb, Numerical approaches to delay equations with small solutions, E. A. Lipitakis(Ed), Proceedings of HERCMA 2001, 101-108.

[5] N. J. Ford, P. M. Lumb, Systems of delay equations with small solutions: A numerical approach, In J. Levesley, I. J. Anderson \& J. C. Mason (Eds), Algorithms for Approximation IV, University of Huddersfield, 2002, 94-101.

[6] N. J. Ford, P. M. Lumb, Mixed-type functional differential equations: a numerical approach (Extended version), Technical Report 2007:3, Department of Mathematics, University of Chester, 2007.

[7] J. Härterich, B. Sandstede, A. Scheel, Exponential Dichotomoies for Linear Non-autonomous Functional Differential Equations of Mixed Type, Indiana University Mathematics Journal, 51, (2002), 1081-1109.

[8] V. Iakovleva, C. J. Vanegas, On the solution of differential equations with delayed and advanced arguments, Electronic Journal of Differential Equations: Conference 13, (2005), 57-63.

[9] T. Krisztin, Nonoscillation for Functional Differential Equations of Mixed Type, Journal of Mathematical Analysis and Applications, 245 (2000), 326-345.

[10] P. M. Lumb, Delay Differential Equations: Detection of Small Solutions, PhD Thesis, University of Liverpool, UK, 2004.

[11] J. Mallet-Paret, The Fredholm Alternative for Functional Differential Equations of Mixed Type, Journal of Dynamics and Differential Equations, 11, (1999), $1-47$.

[12] J. Mallet-Paret, S. M. Verduyn Lunel, Mixed-type functional differential equations, holomorphic factorization, and applications, Proceedings Equadiff 2003, International Conference of Differential Equations, Hasselt 2003, World Scientific, Singapore, (2005) 73-89, ISBN 9812561692.

[13] A. Rustichini, Functional Differential Equations of Mixed Type: The Linear Autonomous Case, Journal of Dynamics and Differential Equations 1, (1989), 121-143.

[14] A. Rustichini, Hopf Bifurcation for Functional Differential Equations of Mixed Type, Journal of Dynamics and Differential Equations 1, (1989), 145-177.

[15] S. M. Verduyn Lunel, About Completeness for a Class of Unbounded Operators, J. Differential Equations 120 (1995), 108-132. 